\title{
Upper facial surgery: simultaneous hairline-lowering surgery during endoscopic forehead lifting
}

Jang Hwan Min ${ }^{1}$, Jinwoo Park², Wooyeol Baek ${ }^{2}$

${ }^{1}$ Batang Plastic Surgery Clinic, Seoul; ${ }^{2}$ Department of Plastic and

Reconstructive Surgery, Institute for Human Tissue Restoration, Severance Hospital, Yonsei University College of Medicine, Seoul, Korea

\begin{abstract}
Background Endoscopic forehead lifting is one of the most common procedures in the field of upper facial surgery. The upper third of the face determines the facial expression and plays a key role in the appearance of facial youth. After the forehead, a high hairline is one of the most important features of the upper third of the face contributing to age identification. The combined evaluation of these two features should be a basic premise of upper facial surgery.

Methods The authors present a surgical sequence in which endoscopic forehead lifting and lowering of the high hairline by means of a scalp flap advancement are carried out during the same operation. The incision line is located along the hairline. After the scalp and forehead flap are moved, they are fixed using the bone tunnel fixation method.

Results In total, 194 patients were treated with endoscopic forehead lifting and simultaneous hairline lowering between August 2018 and July 2020. On average, the patients' hairlines were $18 \mathrm{~mm}$ lower and their eyebrows were $5 \mathrm{~mm}$ higher. No patients had serious complications.

Conclusions Endoscopic forehead lifting and simultaneous hairline lowering surgery make it possible to address the entire upper third of the face in a single facial rejuvenation operation.
\end{abstract}

Keywords Forehead / Lifting / Endoscopy / Hair / Esthetics

\section{INTRODUCTION}

Standards of beauty have changed over time, and the standard of facial beauty is of particular interest. Unlike other parts of the body, the face represents a person's identity and forms a distinct structure that makes oneself distinguishable from other people. Attempts have been made to evaluate the standards of an aesthetically pleasing face, and various measurement methods have been suggested.

Received: Dec 1, 2020 Revised: Apr 15, 2021 Accepted: Jun 14, 2021 Correspondence: Wooyeol Baek Department of Plastic and Reconstructive Surgery, Institute for Human Tissue Restoration, Severance Hospital, Yonsei University College of Medicine, 50 Yonsei-ro, Seodaemun-gu, Seoul 03722, Korea

Tel: +82-2-2228-2220, Fax: +82-2-393-6947, E-mail: PARANDE@yuhs.ac

Copyright @ 2021 The Korean Society for Aesthetic Plastic Surgery.

This is an Open Access article distributed under the terms of the Creative Commons Attribution Non-Commercial License (https://creativecommons.org/licenses/by-nc/4.0/) which permits unrestricted non-commercial use, distribution, and reproduction in any medium, provided the original work is properly cited. www.e-aaps.org
Nonetheless, despite changes in beauty standards, the proportions and symmetry of the face have always been valued. It is important for each part of the face to be in a harmonious proportion to have an aesthetic balance [1]. One of the most effective methods used to assess the balance of facial proportions is to evaluate the artist's canon of "equal facial thirds" [2]. This method measures the ratio of three parts of the face (forehead, nasal segment, and lower segment) in a profile view of the face, and each part should account for roughly one-third of the total length of the face. Similar proportions were also observed in a study involving South Korean women [3].

If the forehead is long, the hairline is positioned upwards and backwards, resulting in a masculine and aged appearance [4]. For women, a genetic predisposition is the primary cause of forehead elongation, although it can also be caused by forehead lift surgery [5]. Women with elongated foreheads either opt for nonsurgical solutions, such as having bangs and tattoos, or undergo surgical correction of the long forehead, such as hair transplantation and forehead reduction. 
If a long and wide forehead is combined with aging of the upper face, mild ptosis, or a narrow lid-brow distance, a forehead lift should be concurrently performed to address these accompanying conditions. Herein, we introduce a method of simultaneously performing hairline-lowering surgery and endoscopic forehead lift through a single hairline incision.

\section{METHODS}

We retrospectively analyzed the medical records of patients who underwent simultaneous hairline lowering surgery during endoscopic forehead lift from August 2018 to July 2020 at our clinic. This study was approved by Yonsei University Severance Hospital, Institutional Review Board (IRB No. 4-2020-1002). Informed consent was also provided by patients. Preoperative evaluations, including a physical examination and review of the patient's medical history, were performed. We recorded whether the patient had undergone previous hair transplantation, semipermanent tattooing, or procedures through a coronal approach. Individuals who had undergone previous procedures via a coronal approach and those with androgenic alopecia were excluded from this study. The patients returned to the clinic for follow-up at 1, 3, 6, and 12 months postoperatively. At these visits, their clinical progress was evaluated, and photographs were obtained using a digital camera (EOS 700D; Canon Corp., Tokyo, Japan). The photographs were captured in a standardized manner by physicians, ensuring that the
Frankfort horizontal plane was clearly visible. To obtain objective values for surgical results, we evaluated the frontal, left and right three-quarters, and crown view photographs. The preoperative clinical evaluation included an examination of the hair direction, frontotemporal points, recession of the anterior hairline, laxity of the scalp, and brow ptosis. The amount of scalp laxity was determined by placing a thumb on the anterior hairline, stretching the skin on the scalp to the greatest possible extent, and measuring the distance that the anterior hairline moved. The forehead length and lid-brow distance were measured pre- and post-surgery to evaluate the surgical outcomes and occurrence of complications. Forehead reduction was measured as the change in forehead length at the middle frontal line. Brow lift was defined as the change in lid-brow distance at the pupil line.

The forehead length of the patient was measured as the distance from the trichion to the nasion. The extent of scalp laxity and brow ptosis was assessed to determine the excess forehead width that needed to be excised. Scalp laxity was measured as the distance of vertical movement elicited by placing the thumb on the anterior hairline and pushing it in the caudal direction as much as possible. The extent of brow ptosis was measured as the amount of elevation that resulted from placing the thumb firmly at approximately $3 \mathrm{~cm}$ above the eyebrow and gently pushing upward.

The surgical procedure is shown in Fig. 1. The operation was performed under monitored anesthesia, and the incision lines were designed with the patient lying in a supine position. An incision
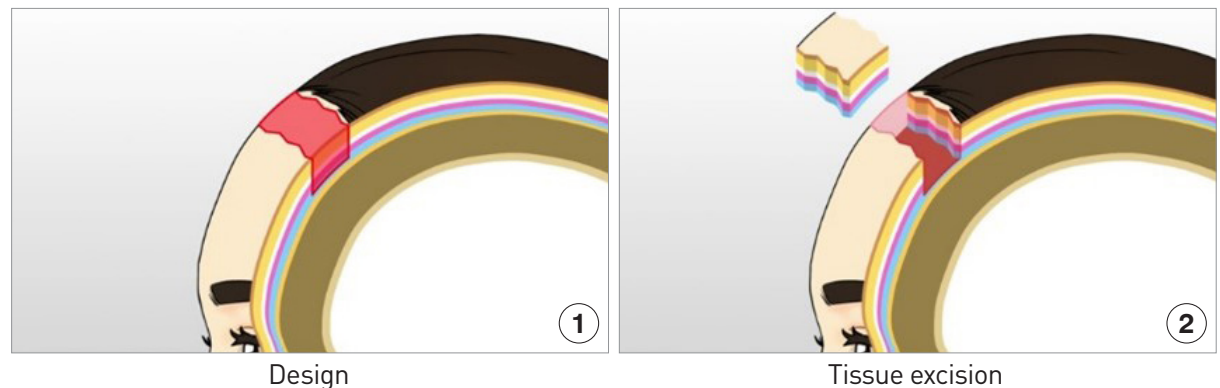

Tissue excision

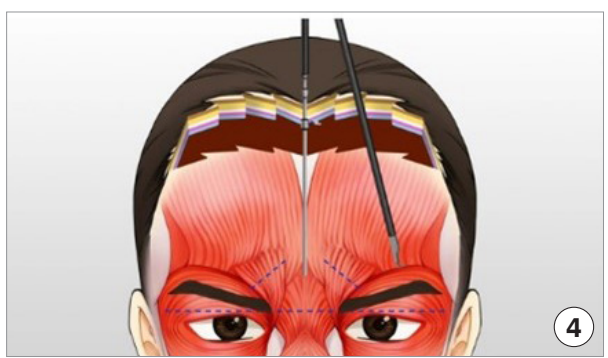

Periosteal tearing

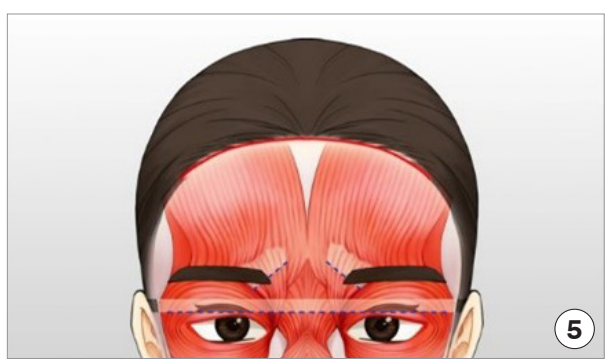

Flap approximation \& fixation

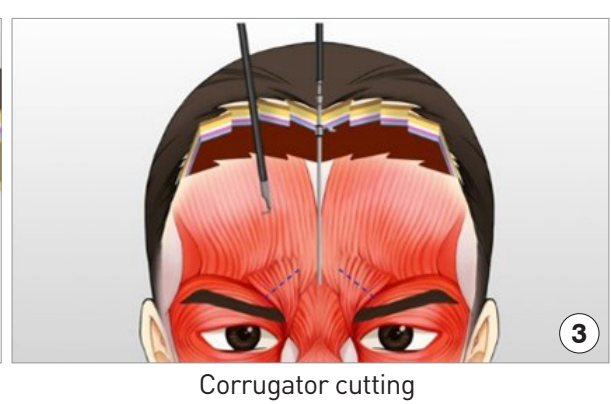

(3)

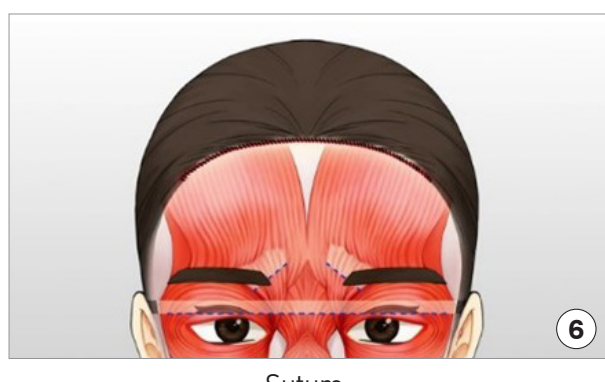

Suture

Fig. 1. Surgical procedure. (1) An incision line was drawn along the hairline, followed by another incision line parallel to the hairline in front of the width of the forehead to be reduced. (2) The entire thickness of the tissue was excised. (3) Using an endoscope through the incision, the corrugators were excised. (4) The periosteum under the eyebrows was dissected horizontally while preserving the supraorbital nerves. (5) The scalp and forehead flaps were moved bidirectionally and approximately fixed at the desired position. (6) Finally, a subcutaneous suture and skin suture were done. 
line was marked that ran parallel to the hairline several millimeters behind the frontmost hair for a trichophytic incision and was designed to form an irregularly irregular line for a natural hairline. Another parallel incision line was then marked anterior to the first incision line, at a distance equaling the sum of the amount of brow ptosis and scalp laxity measured preoperatively. This second incision line became the neo-hairline after surgery. After finishing the design, $1 \%$ lidocaine solution containing epinephrine (1\% lidocaine solution with 1:200,000 epinephrine) was widely injected into the superior orbital rim and incision region. For a trichophytic hairline incision, the beveled incision began 2 to 3 hairs from the transition point, between the lanugo and thicker, dense hair. The incision was beveled to transect the hair shafts. This method helps disguise the scar by inducing the hair to grow through the surgical scar after surgery [6]. Scalp dissection was performed widely from the vertex to the pterion through the sub-galea aponeurotic layer. Forehead dissection was mainly performed in the subperiosteal layer. After making an incision into the periosteum with reference to the lateral orbital rim and the temporal line, the dissection was extended to the pterion in the sub-galea aponeurotic layer. Taking care to preserve the supraorbital and supratrochlear neurovascular bundles through an endoscope, periosteum tearing was performed at the supraorbital rim, and the corrugator muscle was cut to move the forehead flap without tension. An incision was made along the line designed to run anteriorly parallel to the hairline incision, and fullthickness forehead tissue was removed. After removing the periosteum from the area assigned to bone tunneling, seven to nine Vshaped bone tunnels were created using a $1.2-\mathrm{mm}$ burr between the temporal lines on both sides. A 2-0 polydioxanone (PDS) suture engaging the galea layer of the scalp flap and the periosteal layer of the forehead flap was passed through the bone tunnel until the flap was advanced to the desired position, and the suture was tied. Meticulous skin approximation of the scalp flap and forehead flap was performed through a subcutaneous suture using 4-0 PDS. A drain tube was not placed following the procedure, and a running suture was performed using 6-0 nylon for skin closure. To prevent hematoma on the forehead, a slight pressure dressing was applied
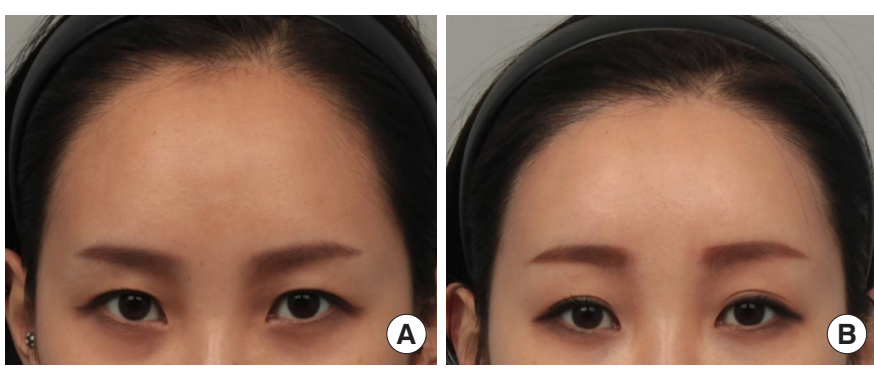

Fig. 2. A 31-year-old woman underwent surgery for the purpose of long forehead correction and to achieve a gentler impression. (A) Before surgery and $(B) 6$ months after surgery. using an elastic bandage. On the third postoperative day, after removing the dressing and applying wound healing ointment, nonstress activities could be resumed. Skin sutures were removed 8 to 9 days after surgery.

The outcome of forehead reduction was measured as the change in forehead length at the middle frontal line. The amount of brow lift was indicated and assessed as the change in lid-brow distance at the pupil line. Statistical analyses were performed using statistical functions in Microsoft Excel (Microsoft Corp., Redmond, WA, USA). Continuous variables were characterized as mean and standard deviation or as median and range. Quantitative variables at different time points were compared using the paired t-test. Statistical significance was defined as $\mathrm{P}<0.05$.

\section{RESULTS}

From August 2018 to July 2020, 194 patients underwent the procedure described herein, of whom 88 patients received more than 2 months of follow-up. These patients comprised 80 women and eight men; their mean age was $33.23 \pm 9.06$ years (range, 22-62 years) and the average follow-up period was $4.17 \pm 2.53$ months (range, $2-17$ months). The average amount of scalp advancement was $17.67 \pm$ $1.78 \mathrm{~mm}$ and the average amount of brow lifting was $5.03 \pm 0.71$ $\mathrm{mm}$. The length of the upper face, which decreased by $15.40 \pm 4.62$ mm compared to the preoperative measurements, was well maintained after surgery, as well as the forehead lift effect; specifically, the eyelid-brow distance was improved by $4.78 \pm 0.94 \mathrm{~mm}$, giving a gentler impression. There was also improvement in hooding of the upper lid and wrinkles on the nasal dorsum. Compared to the preoperative forehead length, the postoperative forehead length was significantly reduced $(\mathrm{P}<0.001)$. The lid-eyebrow distance increased postoperatively, and this change was statistically significant $(\mathrm{P}<0.001)$. Forehead and scalp numbness developed after surgery, but started to disappear in most patients by 2 months after surgery, and sensation returned to normal after an average of 5-6 months. Other complications such as infection, hematoma, and hair loss did not occur (Figs. 2, 3).
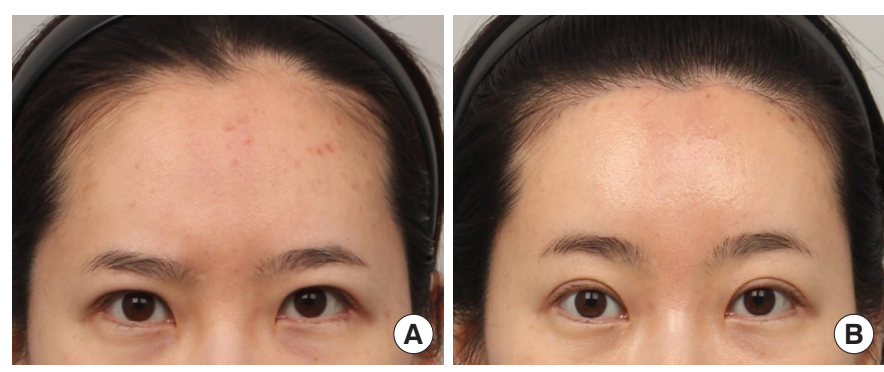

Fig. 3. A 31-year-old woman underwent surgery to correct a short eyebrow distance, brow and double-fold asymmetry, and a long and wide forehead. (A) Before surgery and (B) 4 months after surgery. 


\section{DISCUSSION}

Research on facial rejuvenation has mostly focused on the position of eyebrows and wrinkles on the forehead in the upper third of the face. However, due to the current trend of preferring small faces, the demand for high hairline correction is increasing. Patients with wide foreheads caused by a high hairline have an unbalanced vertical third, which makes them appear older and more masculine. Hair transplantation is the focus of most methods for high hairline correction. Hair transplantation, in which hair follicles harvested from a donor site are implanted one by one to a recipient site, is known as the gold standard for hairline correction. However, this method is labor-intensive for the surgeon and costly and time-consuming for the patient. In addition, it takes at least several months until the patients can see the final results of surgery, and satisfaction with the results is low if adequate hair density is not obtained due to the low survival rate of the transplanted hair. By contrast, hairline-lowering surgery using scalp flap advancement takes relatively little time and has the advantage of correcting forehead elongation in a single session. In addition, the risk of scarring can be reduced by using a trichophytic incision, as hair growth through an incision scar can be expected.

Guyuron and Rowe [5] and Ramirez et al. [7] reported surgical methods for correcting a high hairline. In both studies, surgery was performed by advancing the scalp flap after galea dissection. To prevent reverse movement of the flap, Guyuron used a bone tunneling method and Ramirez fixed the scalp flap with a fixation device (Endotine; Coapt Systems, Inc., Palo Alto, CA, USA).

We used the bone tunneling fixation method to fix the scalp flap and forehead flap. The bone tunnels were created in the outer cortex of the skull using a guard devised to prevent penetration of the inner cortex. As the mean thickness of the frontal and parietal bones where the bone tunnels were made is $3-6 \mathrm{~mm}$ [8], the guard device was designed to prevent the burr tip from advancing more than 5 $\mathrm{mm}$ to prevent penetration of the inner cortex (Fig. 4).

Previously introduced surgical procedures performed in a similar way to ours include forehead reduction $[2,7,9]$ and forehead or brow lift surgery [10-12], which are done through a hairline incision. Some studies introduced a method of bidirectionally approximating the scalp flap and forehead flap after dissection, but unlike our method, the moved flaps were not fixed to the floor before approximation and suturing in those studies $[13,14]$. Our method is different from existing methods in that each flap is fixed to the base through bone tunnel fixation in order to exactly predict the effect of simultaneous forehead reduction and brow lift surgery performed through a single hairline incision.

When hairline-lowering surgery and forehead lift are performed simultaneously using our method, a larger amount of tissue can be excised from the forehead than when each operation is performed separately, and additional incisions and scarring can be prevented

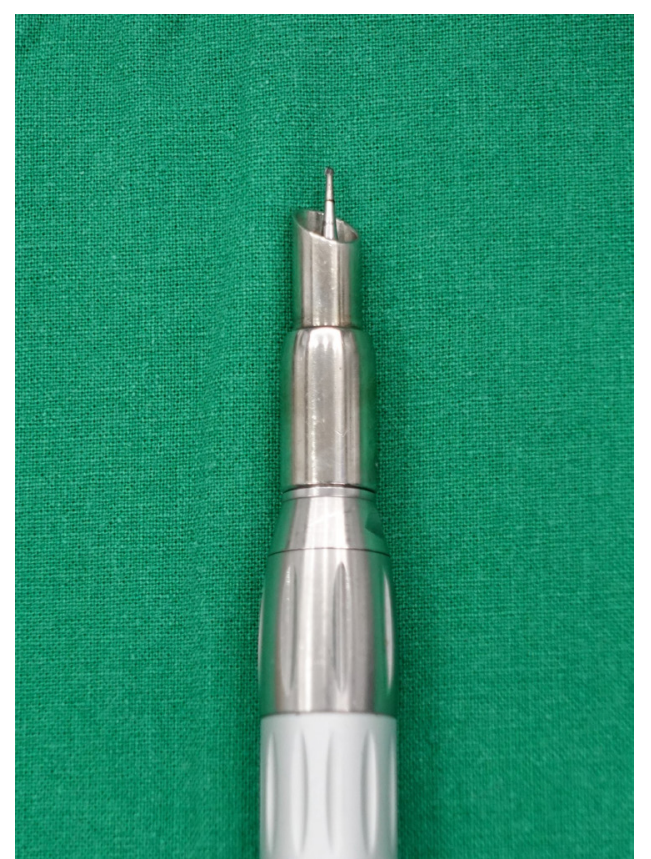

Fig. 4. Guard device that protects the inner cortex of the skull from being penetrated.

by sharing a single incision line. In addition, approximation of the scalp flap and forehead flap on both sides reduces the tension on the incision, which in turn lowers the risk of scar widening. Furthermore, by inducing hair growth through the trichophytic incision, the visibility of the scar can be diminished.

After surgery, patients may experience numbness, swelling, and bruising. Swelling and bruising take roughly a week to disappear. Numbness progresses over 2-6 months, after which sensation returns and most patients experience an itching, tingling, or stinging sensation at approximately 2-3 months after surgery. The appearance of numbness in the early postoperative period seems to be the consequence of nerve bundles being stretched during the advancement of the flaps. Even if there is no direct damage to the nerves, the function of the nerves could be weakened during the procedure. It is known that there is a possibility of transient hair loss due to tension on the scalp caused by advancement of the scalp flap, but no cases of transient hair loss occurred during follow-up. In addition, accumulation of blood under the dissection flap during surgery may lead to hematoma formation. However, hematoma can be prevented by irrigating the area after flap dissection and applying an elastic bandage for 2-3 days, which also contributes to reducing swelling and bruising after surgery. The scars gradually became paler over 3-6 months postoperatively. For scars visible even after 6 months, fractional laser therapy was performed. In one case, scar revision was performed, as fractional laser therapy did not improve the appearance of the scar.

Our method was effective in reducing the length and width of 
the forehead, creating a gentle and bright impression, and making double-fold lines more prominent without eyelid surgery by adjusting the distance between the eyes and eyebrows and the angle of the eyebrows. Further research into the quantitative relationship between the amount of brow lift and the change in fold height is needed to accurately predict surgical outcomes.

\section{NOTES}

\section{Conflict of interest}

No potential conflict of interest relevant to this article was reported.

\section{Ethical approval}

The study was approved by the Institutional Review Board of Yonsei University Severance Hospital (IRB No. 4-2020-1002) and performed in accordance with the principles of the Declaration of Helsinki.

\section{Patient consent}

The patients provided written informed consent for the publication and the use of their images.

\section{ORCID}

Jang Hwan Min

https://orcid.org/0000-0001-9223-0206

Wooyeol Baek

https://orcid.org/0000-0002-6638-4110

\section{REFERENCES}

1. Freilinger G. Proportions of the aesthetic face. Acta Chir Austriaca 1985;17:61.
2. Marten TJ. Hairline lowering during foreheadplasty. Plast Reconstr Surg 1999;103:224-36.

3. Lee JS, Kim HK, Kim YW. Anthropometric analysis of the attractive and normal faces in Korean female. J Korean Soc Plast Reconstr Surg 2004;31:526-31.

4. Klatsky SA. Forehead plasty for facial rejuvenation. Aesthet Surg J 2000; 20:416-26.

5. Guyuron B, Rowe DJ. How to make a long forehead more aesthetic. Aesthet Surg J 2008;28:46-50.

6. Kim JM, Hong JG. Endoscope-assisted trichophytic anterior hairline brow lift. Arch Aesthetic Plast Surg 2016;22:144-8.

7. Ramirez AL, Ende KH, Kabaker SS. Correction of the high female hairline. Arch Facial Plast Surg 2009;11:84-90.

8. Hwang K, Kim JH, Baik SH. The thickness of the skull in Korean adults. J Craniofac Surg 1999;10:395-9.

9. Kabaker SS, Champagne JP. Hairline lowering. Facial Plast Surg Clin North Am 2013;21:479-86.

10. Guyuron B, Gatherwright J, Totonchi A, et al. Cessation of hairline recession following open forehead rejuvenation. Plast Reconstr Surg 2014;133:1e-6e.

11. Owsley TG. Subcutaneous trichophytic forehead browlift: the case for an "open" approach. J Oral Maxillofac Surg 2006;64:1133-6.

12. Spiegel JH. Scalp advancement and the pretrichial brow lift. Facial Plast Surg 2018;34:145-9.

13. Lee Y, Kim J. Dual-vector foreheadplasty for reducing the vertical height of the forehead with concomitant mid-facelift. Arch Aesthetic Plast Surg 2012;18:106-10.

14. Kim JB, Kim SW, Chung JY. New surgical technique: bidirectional forehead narrowing and eyebrow lifting using regional flap mobility. Plast Reconstr Surg Glob Open 2020;8:e2836. 